\title{
Knockdown of FUSE binding protein 1 enhances the sensitivity of epithelial ovarian cancer cells to carboplatin
}

\author{
JINLI ZHANG $^{1 *}$, XIFENG XIONG $^{1 *}$, XING HUA $^{2}$, WENJUAN CAO $^{1}$, SHENGNAN QIN $^{1}$, \\ LIBING DAI $^{1}$, PEIHONG LIANG ${ }^{1}$, HUILING ZHANG ${ }^{3}$ and ZHIHE LIU ${ }^{1}$ \\ ${ }^{1}$ Guangzhou Institute of Traumatic Surgery, ${ }^{2}$ Department of Pathology, Guangzhou Red Cross Hospital, \\ Medical College, Jinan University, Guangzhou, Guangdong 510220; ${ }^{3}$ Department of Pharmacology, \\ College of Pharmaceutical Science, Soochow University, Suzhou, Jiangsu 215123, P.R. China
}

Received April 28, 2016; Accepted July 11, 2017

DOI: $10.3892 / 01.2017 .6978$

\begin{abstract}
Epithelial ovarian cancer (EOC) affects almost 25,000 women annually and is the fifth most common malignancy in women in North America. A combination of surgery and cytotoxic chemotherapy may produce a favorable clinical response. The platinum-paclitaxel combination regimen is the chemotherapy gold-standard for advanced ovarian cancer, and carboplatin is one of the agents in this combination therapy. However, the majority of patients eventually experience a relapse due to the development of platinum resistance. FUSE binding protein 1 (FBP1) has been identified as an anti-apoptotic and pro-proliferative oncoprotein that is overexpressed in hepatocellular carcinoma. Its high expression is also associated with carboplatin resistance. In the present study, it was identified that the expression of FBP1 was significantly higher in EOC tissues than in normal epithelial ovarian or in epithelial ovarian adenoma tissue. FBP1 expression was significantly correlated with the grade of epithelial ovarian cancer. Carboplatin inhibited the expression of FBP1 in epithelial ovarian cancer cells and the knockdown of FBP1 enhanced the inhibition of cell viability and migration by carboplatin. In addition to FBP1, carboplatin also inhibited the expression of $\beta$-catenin and matrix metalloproteinase (MMP)-9. Furthermore, the expression of $\beta$-catenin and MMP-9 were lower in FBP1 knockdown cells compared with control EOC cells. FBP1 may thus serve a role in the regulation of the expression of $\beta$-catenin and MMP-9; the inhibition of $\beta$-catenin and MMP-9 by carboplatin may be mediated through the inhibition of FBP1. The inhibition of
\end{abstract}

Correspondence to: Dr Zhihe Liu, Guangzhou Institute of Traumatic Surgery, Guangzhou Red Cross Hospital, Medical College, Jinan University, 396 Tongfu Zhong Road, Guangzhou, Guangdong 510220, P.R. China

E-mail: zliu0731@163.com

*Contributed equally

Key words: epithelial ovarian cancer, carboplatin, fuse binding protein 1, apoptosis, migration
FBP1 expression by carboplatin may be a mechanism in the treatment of EOC by carboplatin.

\section{Introduction}

Epithelial ovarian cancer (EOC) affects almost 25,000 women annually and is the fifth most common malignancy in women in North America, with a five-year mortality rate of $>70 \%$ (1). As the symptoms may not be observed until the cancer has spread extensively, $<25 \%$ of women are diagnosed in the early stages of the disease. The combination of surgery with cytotoxic chemotherapy produces favorable clinical responses in $50-80 \%$ of patients $(2,3)$.

The platinum-paclitaxel combination regimen is the chemotherapy gold-standard for advanced ovarian cancer, with response rates $>80 \%$ and complete response rates of 40-60\% (4-8). Carboplatin and cisplatin are two agents used in this combination regimen. These compounds share the mechanism of the formation of DNA adducts, and cross-resistance is frequently observed (9). As a result of the DNA adducts, signaling pathways including cell cycle checkpoints, p53 signaling and mitogen-activated protein kinases, are activated, ultimately leading to cell death $(10,11)$. However, the majority of patients eventually experience a relapse even if they respond to platinum-paclitaxel combination treatment in the beginning, with a median progression-free survival time of 18 months (12). The mechanisms implicated in platinum resistance include decreased platinum uptake, enhanced DNA damage repair and increased resistance to apoptosis (9-11). Patients that develop platinum-resistant or refractory disease are treated with a range of other drugs, including paclitaxel, bevacizumab and capecitabine (13). However, improving the rate of curing EOC remains critical. Therefore, it is necessary to search for novel, biologically targeted treatment modalities. The understanding of the molecular biology of cancer, including the mechanisms underlying cancer processes and drug resistance, has facilitated the development of targeted therapies, including small-molecule inhibitors. These agents target proteins associated with malignant cell behavior, including cell viability/death, metastasis and angiogenesis.

Far upstream element (FUSE) binding protein 1 (FBP1) has been identified as an anti-apoptotic and pro-proliferative 
oncoprotein that is overexpressed in hepatocellular carcinoma $(14,15)$. It has also been demonstrated that the high expression of FBP1 is associated with carboplatin resistance (16). FBP1 functions as a transcriptional regulator by binding to the single-stranded DNA element, FUSE, and interacting with the basal transcriptional machinery (17). Target genes regulated by FBP1 include the oncogene c-Myc (18), the cell cycle inhibitor p21 (15) and the deubiquitinating enzyme ubiquitin-specific peptidase 29 (Usp29) (19). Recently, Rabenhorst et al (20) demonstrated that FBP1 additionally serves a role in hematopoietic development and homeostasis. In our previous studies, we identified that FBP1 physically interacts with p53 to suppress p53 transcriptional activity during radiation-induced cellular stress, and that it facilitates hepatitis $\mathrm{C}$ virus replication in hepatoma cells $(21,22)$. Therefore, the present study considered the association of FBP1 expression with EOC progression and the response to carboplatin treatment.

In the present study, it was identified that the silencing of FBP1 enhanced the sensitivity of EOC cells to carboplatin. Additionally, carboplatin treatment inhibited EOC cell viability and migration by inhibiting the expression of FBP1. Therefore, FBP1 may be a novel potential biological target for the treatment of EOC.

\section{Materials and methods}

Clinical tissue samples and immunohistochemical staining (IHS). The study was conducted subsequent to obtaining informed consent from all subjects, and the approval of the study protocol by the Medical Ethics Committee of Guangzhou Red Cross Hospital of Medical College, Jinan University (Guangzhou, China). Samples were collected between January 2012 and June 2015; patients had a median age of 45.5 years (range, 17-76 years). Samples were assigned into three groups, including normal epithelial ovarian tissue (4 samples), epithelial ovarian adenoma tissue (7 samples) and epithelial ovarian cancer tissue (10 samples).

Paraffin sections $(5-\mathrm{mm})$ were deparaffinized in $100 \%$ xylene and rehydrated in a descending series of ethanol-water solutions. The sections were boiled in $10 \mathrm{mmol} / 1$ citrate buffer (pH 6.0) for $20 \mathrm{~min}$ for antigen retrieval. Endogenous peroxidase activity was blocked with $0.3 \%$ methanolic peroxide for $30 \mathrm{~min}$ at room temperature. Anti-FBP1 antibody (cat. no. sc-136137, 1:100) was obtained from Santa Cruz Biotechnology, Inc. (Dallas, TX, USA). Horseradish peroxidase (HRP)-conjugated secondary antibody was also obtained from Santa Cruz Biotechnology (cat. no. sc-2004, 1:100). The antigen-antibody reactions were visualized with the chromogen diaminobenzidine (DAB).

Stained sections were observed under a microscope. DAB density was quantified by a video camera mounted on an optical microscope (Microphot; Nikon Corporation, Tokyo, Japan) connected to a video capture card. Images were captured with a 440 objective and image processing and analyses were performed using Image-Pro Plus 6.0 software (Media Cybernetics, Inc., Shanghai, China). The intensity of the immunohistochemical reaction was expressed as the integrated absorbance (IA) of the DAB reaction product. The results of 5 separate measurements for each sample were expressed as the mean \pm standard deviation (SD).
Cell culture. SKOV3 ovarian cancer cells were obtained from the American Type Culture Collection (Manassas, VA, USA). The cells were grown in complete Dulbecco's modified Eagle's medium (DMEM) containing 10\% fetal bovine serum (FBS.), $100 \mathrm{U} / \mathrm{ml}$ penicillin, $100 \mu \mathrm{g} / \mathrm{ml}$ streptomycin (Thermo Fisher Scientific, Inc., Waltham, MA, USA) and kept at $37^{\circ} \mathrm{C}$ in a humidified atmosphere with $5 \% \mathrm{CO}_{2}$.

Construction of FBP1 knockdown lentivirus and generation of stable FBPl knockdown cells. A pSi-LVRH1GP vector with a puromycin resistance cassette (GeneCopoeia, Rockville, MD, USA) was used to express short hairpin (sh)RNA to knock down FBP1 expression. The control vector expressed a scrambled sequence (5'-GCTTCGCGC CGTAGTCTTA-3') and was designated pSi-LV-FBP1-C. To knock down FBP1, several shRNA sequences were used, including the sequences from 1036-1056 (5'-GGACAACAC CCGAAAGGATAG-3'), 1671-1671 (5'-GCAGGAACGGAT CCAAATTCA-3') and 1758-1778 (5'-GCAGGTGCACCA ACTACAACT-3') of FBP1; these vectors were designated as pSi-LV-FBP1-KD. SKOV3 cells were transfected with pSi-LV-FBP1-C or pSi-LV-FBP1-KD. First, $2 \times 10^{5}$ SKOV3 cells were seeded in $2 \mathrm{ml}$ antibiotic-free DMEM medium supplemented with FBS and cells were incubated until they reached 60-80\% confluence. After washing the cells once with $2 \mathrm{ml}$ of antibiotic- and FBS-free DMEM medium, $900 \mathrm{ul}$ antibiotic- and FBS-free DMEM medium was added to the cells, alongside $1 \mu \mathrm{g}$ of pSi-LV-FBP1-C or pSi-LV-FBP1-KD diluted in $100 \mathrm{ul}$ antibiotic and FBS-free DMEM medium. After $6 \mathrm{~h}$, medium was changed for DMEM supplemented with FBS and antibiotics. Puromycin was used as a selective marker. After $48 \mathrm{~h}$ incubation, medium was changed to DMEM growth medium containing $1.5 \mu \mathrm{g} / \mathrm{ml}$ of puromycin (Santa Cruz Biotechnology, Inc.). Western blotting was used to confirm the knockdown. The pSi-LV-FBP1-C-transfected cells were designated FBP1-C cells, and the cells with the most complete FBP1 knockdown as FBP1-KD cells.

Cell viability assay. SKOV3 FBP1-C or FBP1-KD cells were seeded at $2.0 \times 10^{4}$ cells/well in a 24 -well plate overnight. They were then treated with a range of concentrations of carboplatin (including 0, 38, 77, 134 and $269 \mu \mathrm{mol} / \mathrm{l}$ ) for $48 \mathrm{~h}$. Cell viability was determined by the inner salt (MTS) method, according to the Cell Titer 96 Aqueous One Solution Viability assay manual (Promega Corporation, Madison, WI, USA). The experiment was repeated at least three times.

Western blot analysis. Whole-cell extracts were prepared with radioimmunoprecipitation assay buffer $[150 \mathrm{mM} \mathrm{NaCl}$, $50 \mathrm{mM}$ Tris (pH 7.4), 1\% NP40, 0.1\% SDS and $0.5 \%$ sodium deoxycholate] supplemented with $10 \mathrm{mmol} / \mathrm{l}$ phenylmethanesulfonyl fluoride (Amresco, LLC, Solon, OH, USA). The concentration of protein was determined with a bicinchoninic protein assay kit (Bio-Rad Laboratories, Inc., Hercules, CA, USA). A total of $30 \mu \mathrm{g}$ protein per lane were separated by $10 \%$ SDS-PAGE gel and electro-blotted onto nitrocellulose membranes (Bio-Rad Laboratories, Inc.). The membranes were blocked with $5 \%(\mathrm{w} / \mathrm{v})$ skimmed milk powder in Tris-buffered saline with Tween-20 [TBST; $10 \mathrm{mM}$ Tris (pH 7.5), $150 \mathrm{mM}$ $\mathrm{NaCl}, 0.1 \%$ (v/v) Tween-20]. The membranes were then 

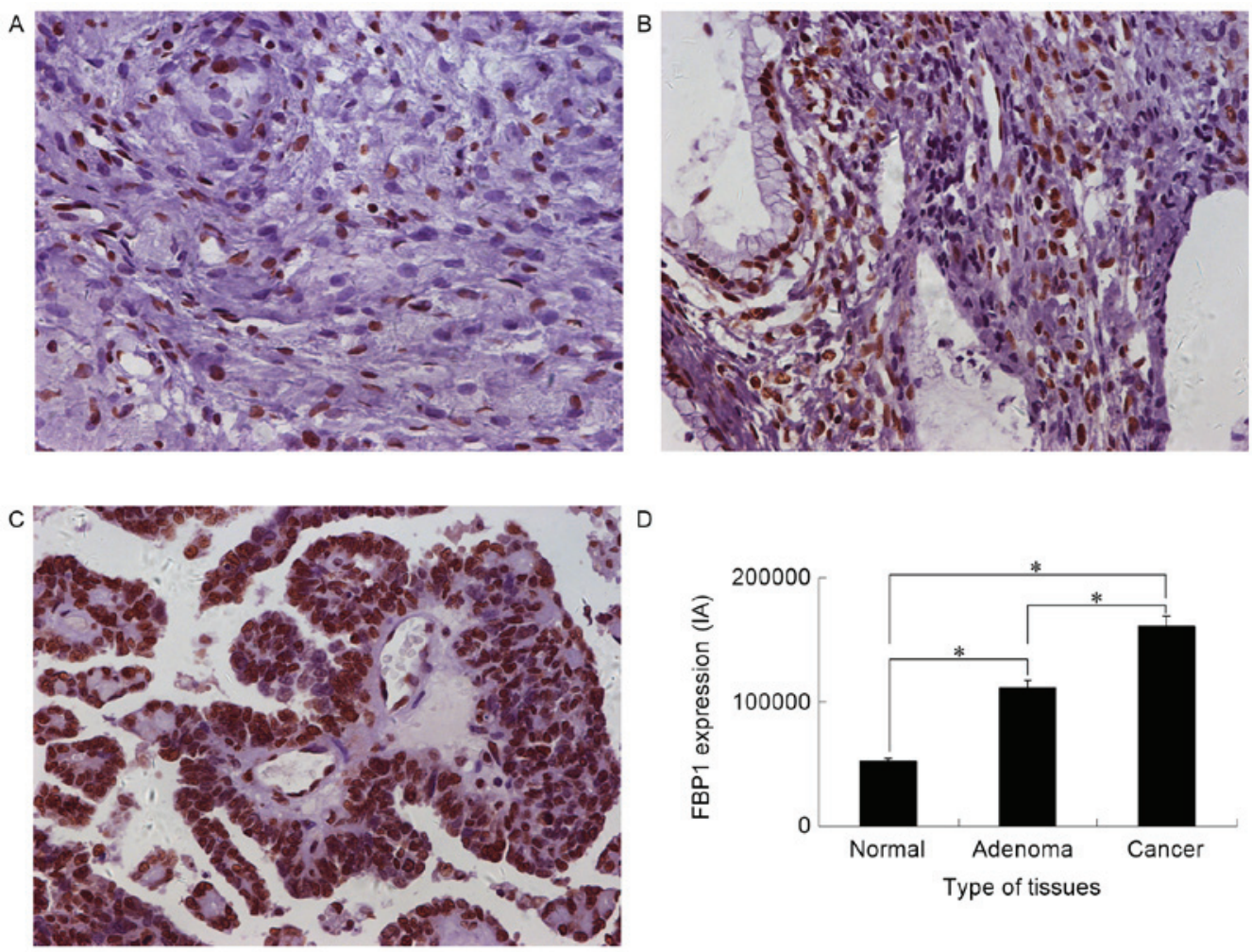

Figure 1. FBP1 expression in epithelial ovarian tissues as determined by immunohistochemical staining. Representative images of FBP1 expression in (A) normal epithelial ovarian tissue, (B) epithelial ovarian adenoma tissue and (C) epithelial ovarian cancer tissues. (D) Quantitative analysis of FBP1 expression in normal epithelial ovarian, epithelial ovarian adenoma and epithelial ovarian cancer tissue (magnification, $\mathrm{x} 400$ ). The intensity of the diaminobenzidine reaction product was expressed as IA. "P<0.05. FBP1, FUSE binding protein 1; IA, integrated absorbance.

incubated at $4^{\circ} \mathrm{C}$ overnight with antibodies against FBP1 (Santa Cruz Biotechnology, Inc.; cat. no. sc-136137; 1:200), $\beta$-catenin (Santa Cruz Biotechnology, Inc.; cat. no. sc-7963; 1:200), cleaved caspase-3 (Cell Signaling Technology, Inc., Danvers, MA, USA; cat. no. 9664; 1:1,000) or matrix metalloproteinase (MMP) 9 (Santa Cruz Biotechnology, sc-21733; 1:200) and GAPDH (Cell Signaling Technology, Inc.; cat. no. $5174 ; 1: 1,000)$ in $5 \%(\mathrm{w} / \mathrm{v})$ skimmed milk powder in TBST. Subsequent to washing three times in TBST, membranes were incubated with HRP-conjugated secondary antibodies (Cell Signaling Technology, Inc.; cat. nos. 7076 and 7074) diluted in TBST $(1: 5,000)$ for $1 \mathrm{~h}$ at room temperature. Subsequent to washing a further three times with TBST, the target proteins were detected using an ECL Western Blotting Substrate kit (Thermo Fisher Scientific, Inc.; cat. no. 32106) and quantified with a ChemiDoc $\mathrm{XRS}^{+}$Imaging system (Bio-Rad Laboratories, Hercules, CA, USA).

Migration assay. SKOV3 FBP1-C or FBP1-KD cells were grown to confluence in 6 -well plates pre-coated with $0.1 \%$ gelatin, then incubated with $10 \mu \mathrm{g} / \mathrm{ml}$ mitomycin C (both Sigma-Aldrich; Merck KGaA, Darmstadt, Germany) for $2 \mathrm{~h}$ to inactivate cell viability. The cell monolayer was wounded with a pipette tip and washed with PBS. DMEM supplemented with $1 \%$ FBS was added into the wells with $5 \mathrm{ng} / \mathrm{ml}$ vascular endothelial growth factor (VEGF; R\&D Systems, Minneapolis, MN, USA) or $5 \mathrm{ng} / \mathrm{ml}$ VEGF with 38 or $77 \mu \mathrm{mol} / 1$ carboplatin. Images were captured at $48 \mathrm{~h}$. The migration distance of the cells was quantified as wound width at $0 \mathrm{~h}$-wound width at $48 \mathrm{~h}$. Three independent experiments were performed.

Statistical analysis. Data are presented as the mean \pm SD. Statistical analysis was performed using SPSS 18.0 software (SPSS, Inc., Chicago, IL, USA). A Student's t-test was performed to test statistical significance between groups. A nonparametric Spearman's rank correlation analysis was performed to evaluate the correlation between FBP1 expression and increasing EOC grade. $\mathrm{P}<0.05$ was considered to indicate a statistically significant difference.

\section{Results}

The expression of FBP1 in epithelial ovarian tissue. To explore the potential role of FBP1 in EOC development, the expression of FBP1 in normal epithelial ovarian, epithelial ovarian adenoma and advanced EOC tissue was analyzed with IHS. FBP1 expression was identified in all three groups (Fig. 1). The FBP1 IA was 52,060 $\pm 27,749$ for normal epithelial ovarian, 111,770 $\pm 44,299$ for epithelial ovarian adenoma and $161,067 \pm 58,531$ for EOC tissue. Significant differences in IA were identified between normal epithelial ovarian tissue and epithelial ovarian adenoma or EOC tissue, and between epithelial ovarian adenoma and EOC tissues $(\mathrm{P}<0.05)$. The highest FBP1 expression was identified in EOC tissue (Fig. 1D). FBP1 expression was positively correlated with the epithelial ovarian grade, as analyzed by a Spearman's rank correlation analysis; the correlation coefficient was $0.637(\mathrm{P}<0.001)$. 

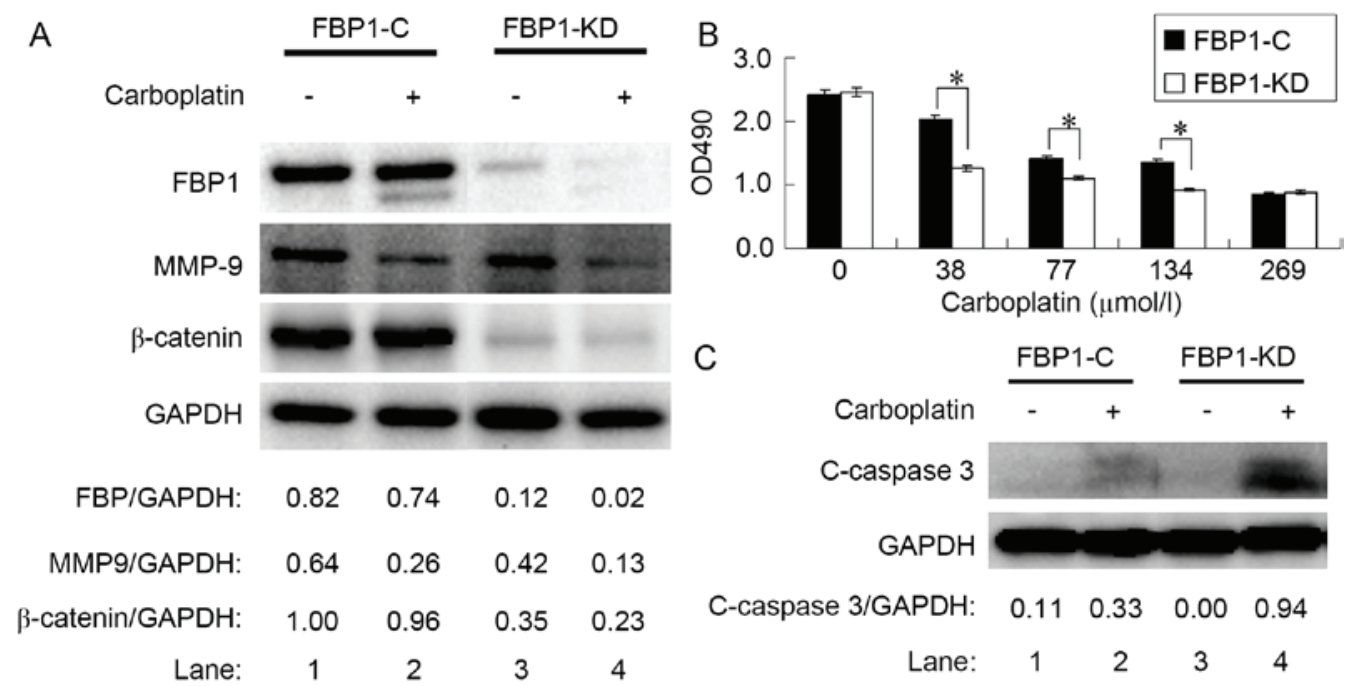

Figure 2. Knockdown of FBP1 enhanced the sensitivity of SKOV3 cells to carboplatin. (A) Protein expression levels of FBP1, MMP-9 and $\beta$-catenin relative to GAPDH, as determined by western blotting, compared between FBP1-C and FBP1-KD cells with, or without, carboplatin treatment. (B) Relative viability of SKOV3 cells treated with carboplatin for $48 \mathrm{~h}$ as determined by an MTS assay. (C) Protein expression level of c-caspase 3 relative to GAPDH, as determined by western blotting, compared between FBP1-C and FBP1-KD cells with, or without, carboplatin treatment. "P<0.05. FBP1, FUSE binding protein 1; MMP-9, matrix metalloproteinase-9; FBP1-C, SKOV3 cells stably transfected with control vector; FBP1-KD, SKOV3 cells stably transfected with FBP1 knockdown vector; c-caspase 3 , cleaved caspase 3.

The generation of ovarian cancer cells with stable FBPI knockdown. SKOV3 cells were transfected with pSi-LV-FBP1-C or -KD vectors to generate FBP1-C and FBP1-KD SKOV3 cells. The interference rate, as analyzed by RT-qPCR, was $57 \%$ for sequence $1,036-1,056,76 \%$ for $1,671-1,691$ and $56 \%$ for $1,758-1,778$. The result was confirmed by western blot analysis (Fig. 2A, lane 1 and 3); thus, cells knocked down with sequence 1671-1691 were used for further experiments in the study. The protein expression of FBP1 in FBP1-KD cells was $\sim 15 \%$ of FBP1-C cells.

FBP1 knockdown increases the sensitivity of ovarian cancer cells to carboplatin. To assess the cytotoxicity of carboplatin, the inhibitory effect on FBP1-C and FBP1-KD SKOV3 cell viability was assessed with an MTS assay. Carboplatin treatment significantly inhibited the viability of FBP1-C and FBP-KD SKOV3 cells from $38 \mu \mathrm{mol} / 1$ (Fig. 2B). The knockdown of FBP1 increased the sensitivity of SKOV3 cells to carboplatin. The decrease in cell viability following carboplatin treatment was significantly enhanced by FBP1 knockdown in the range of 38-134 $\mu \mathrm{mol} / 1$ carboplatin $(\mathrm{P}<0.05)$.

Carboplatin downregulates FBP1 and $\beta$-catenin, and stimulates cleaved-caspase-3 expression. As the knockdown of FBP1 increased the sensitivity of SKOV3 cells to carboplatin, the level of FBP1 expression in SKOV3 cells treated with $38 \mu \mathrm{mol} / 1$ of carboplatin for $48 \mathrm{~h}$ was determined. FBP1 expression was decreased by carboplatin treatment in FBP1-C and in FBP1-KD cells, with a greater decrease in FBP1-KD than in FBP1-C SKOV3 cells (Fig. 2A, lanes 2 and 4; $\mathrm{P}<0.05$ ).

It was previously reported that $\beta$-catenin expression was elevated in EOC (23), potentially contributing to the carboplatin resistance of ovarian cancer cells (24). In the present study, the expression of $\beta$-catenin in FBP1-KD SKOV3 cells was decreased relative to FBP1-C (Fig. 2A, lanes 1 and 3). Carboplatin treatment inhibited $\beta$-catenin expression by 4 and
$35 \%$ in FBP1-C and FBP1-KD SKOV3 cells, respectively (Fig. 2A, lanes 2 and $4, \mathrm{P}<0.05$ ).

Cleaved caspase 3 is a marker for apoptosis. Carboplatin treatment increased the expression of cleaved caspase- 3 ; the increase in cleaved caspase-3 expression was more significant in FBP1-KD than in FBP1-C SKOV3 cells following treatment with $38 \mu \mathrm{mol} / \mathrm{l}$ of carboplatin for $48 \mathrm{~h}$ (Fig. $2 \mathrm{C}, \mathrm{P}<0.05$ ). The result indicated that carboplatin induced apoptosis.

Carboplatin inhibits the migration of SKOV3 cells. Cell migration is critical for cancer invasion and metastasis. The effect of carboplatin on the chemotactic motility of FBP1-C and FBP1-KD SKOV3 cells was measured with a wound-healing assay. Carboplatin inhibited VEGF-induced SKOV3 migration in FBP1-C SKOV3 cells in a dose dependent manner (Fig. 3). The inhibition of cell migration by carboplatin was more significant for the FBP1-KD SKOV3 cells, compared with FBP1-C $(\mathrm{P}<0.05)$. The result indicated that FBP1 knockdown facilitated the inhibition of migration by carboplatin.

MMPs promote extracellular matrix degradation and cell migration, so the effects of carboplatin on MMP-9 expression were investigated. FBP1-C and FBP1-KD SKOV3 cells were treated with $38 \mu \mathrm{mol} / \mathrm{l}$ carboplatin for $48 \mathrm{~h}$. Carboplatin inhibited the expression of MMP-9 (Fig. 2A). The MMP-9 decrease in FBP1-C SKOV3 cells was 59\% (Fig. 2A, lanes 1 and 2), whereas the MMP-9 decrease in FBP1-KD SKOV3 cells was $69 \%$ (Fig. 2A, lanes 3 and 4).

\section{Discussion}

In our previous study, it was demonstrated that FBP1 was abundantly expressed in hepatocellular carcinoma tumors with chronic hepatitis $\mathrm{C}$ backgrounds (22). In the present study, the high expression of FBP1 was identified in EOC and it was demonstrated that FBP1 expression was associated with EOC progression. 


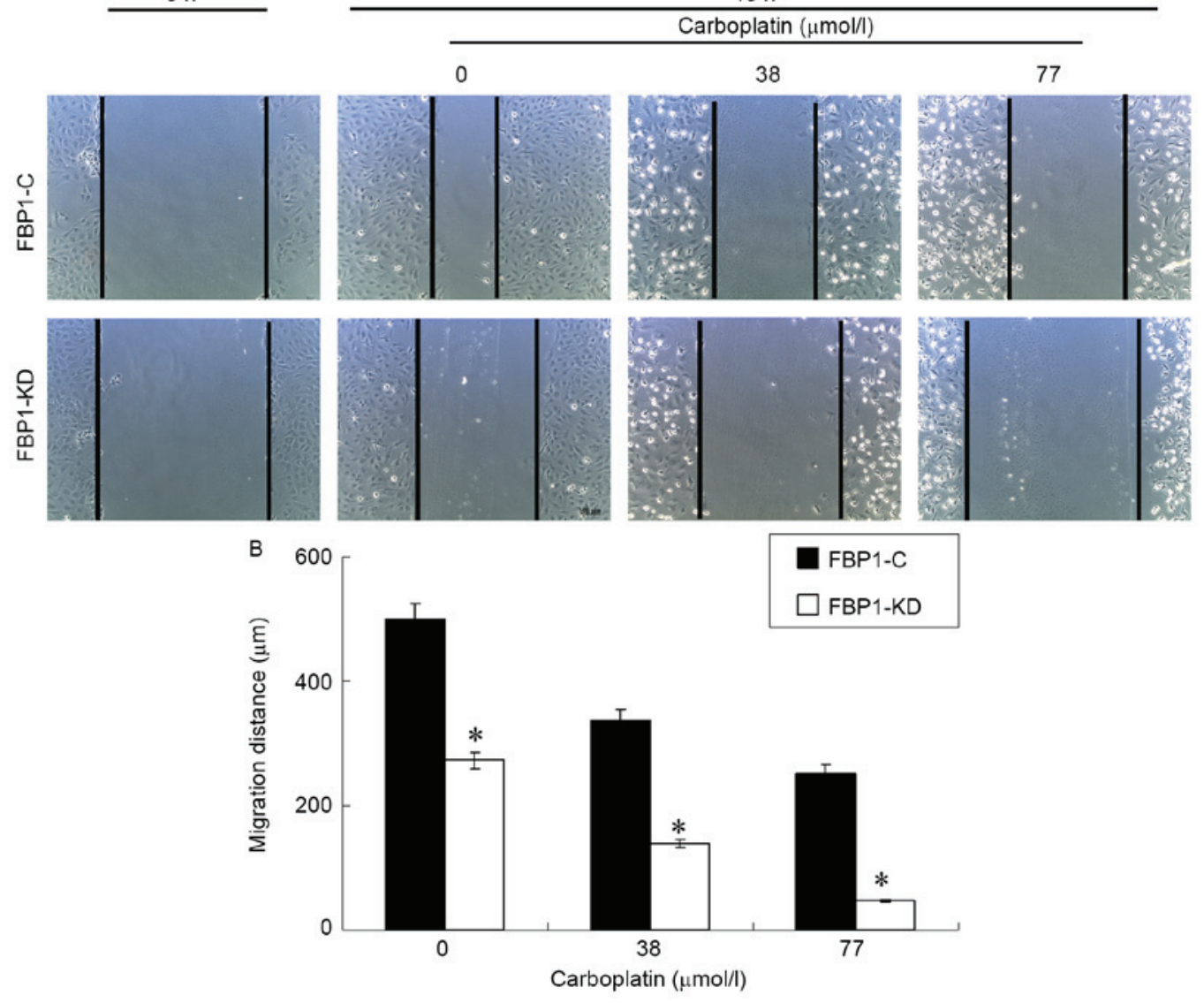

Figure 3. Knockdown of FBP1 enhanced the inhibition of cell migration by carboplatin. (A) Wound healing assay at 0 and 48 h, compared between FBP1-C and FBP1-KD cells with, or without, carboplatin treatment. (B) Quantification of the wound healing assay. The cell migration distance was quantified by the wound width at $0 \mathrm{~h}$ minus the wound width at $48 \mathrm{~h}$. Three independent experiments were performed. * $\mathrm{P}<0.05$. FBP1, FUSE binding protein 1 ; FBP1-C, SKOV3 cells stably transfected with control vector; FBP1-KD, SKOV3 cells stably transfected with FBP1 knockdown vector.

Carboplatin is commonly used to treat EOC (4-9). However, the majority of patients eventually experience a relapse, with a median progression-free survival time of 18 months (12). A recent study in patients with refractory breast cancer demonstrated that tumor profiling-based therapy resulted in a survival benefit (23). Therefore, improving the understanding of the mechanisms implicated in platinum resistance may improve the management of EOC. In our previous study, it was identified that FBP1 knockdown enhances the sensitivity of hepatoma cells to $\gamma$-irradiation (21). In the present study, it was demonstrated that the knockdown of FBP1 enhanced the sensitivity of EOC cells to the growth- and migration-inhibiting effect of carboplatin. This result confirmed the conclusion of a previous study, in which FBP1 was identified as one of eight genes associated with carboplatin resistance, based on the receiver operating characteristics analysis of 1,452 patients (16).

The high expression of $\beta$-catenin was previously identified in EOC (24) and elevated $\beta$-catenin expression may contribute to the carboplatin resistance of ovarian cancer cells (25). The present study identified that the expression of $\beta$-catenin decreased significantly in the cells with FBP1 knockdown and carboplatin treatment inhibited the expression of $\beta$-catenin in FBP1-KN cells to a greater extent than in FBP1-C cells. This result implied that low $\beta$-catenin expression, resulting from FBP1 knockdown, increased the sensitivity of epithelial ovarian cells to carboplatin. Carboplatin treatment also upregulated the expression of cleaved-caspase 3, a marker for apoptosis, in FBP1-KN to a greater extent than FBP1-C cells. Therefore, carboplatin treatment induced a greater extent of apoptosis in FBP1-KN cells than in FBP1-C cells.

MMPs belong to a family of $\mathrm{Zn}^{2+}$-binding, $\mathrm{Ca}^{2+}$-dependent endopeptidases with the essential function of proteolysis of the extracellular matrix, which is associated with several cellular processes (26); MMPs are considered to regulate a number of processes, including cell migration, viability, apoptosis, angiogenesis, tumor expansion and metastasis (27-29). Although they are generally expressed at low levels, MMPs are upregulated during tissue remodeling, inflammation, wound healing and cancer progression $(30,31)$. In the present study, it was demonstrated that carboplatin inhibited EOC cell migration, and that the knockdown of FBP1 enhanced the inhibition of cell migration by carboplatin. Additionally, carboplatin treatment decreased the expression of MMP-9.

Understanding the mechanisms underlying cancer processes, including drug resistance, may facilitate the development of targeted therapies. FBP1 has been identified as an anti-apoptotic and pro-proliferative oncoprotein that is overexpressed in a number of cancer types, including human hepatocellular carcinoma and non-small cell lung cancer (32). It has also been demonstrated that the high expression of 
FBP1 is associated with carboplatin resistance (16). In a recent study, it was reported that $\mathrm{FBP} 2$, another member of the FBP family, regulates doxorubicin resistance in human breast cancer cell lines (33). In addition to regulating the expression of the oncogene c-Myc (18), FBP1 also regulates the expression of p21 (15) and Usp29 (19) and suppresses p53 transcription activity $(21,22)$. In the present study, the expression of $\beta$-catenin and MMP-9 was lower in FBP1-KD SKOV3 cells compared with FBP1-C SKOV3 cells. This may imply that FBP1 serves a role in the regulation of the expression of $\beta$-catenin and MMP-9. It is also reasonable to hypothesize that the effect of carboplatin on the expression of $\beta$-catenin and MMP-9 is mediated through the inhibition on FBP1.

Based on the data in the present study, FBP1 knockdown enhanced the sensitivity of epithelial ovarian cancer cells to carboplatin. FBP1 knockdown enhanced the inhibition of cell viability and migration by carboplatin. Therefore, it is concluded that the high expression of FBP1 is a potential mechanism for carboplatin resistance, and inducing the downregulation of FBP1 is a potential strategy for a novel EOC treatment regimen.

\section{Acknowledgements}

The present study was supported by the National Natural Science Foundation of China (grant nos. 81272222, 30973510, 81171104 and 81473211).

\section{References}

1. A JY, Wang GJ, Sun JG, Gu YC, Wu MS and Liu JH: Identification of phase I and phase II metabolites of Guanfu base A hydrochloride in human urine. Eur J Drug Metab Pharmacokinet 28: 265-272, 2003

2. Berkenblit A and Cannistra SA: Advances in the management of epithelial ovarian cancer. J Reprod Med 50: 426-438, 2005.

3. Salom E, Almeida Z and Mirhashemi R: Management of recurrent ovarian cancer: Evidence-based decisions. Curr Opin Oncol 14: 519-527, 2002.

4. Agarwal R and Kaye SB: Ovarian cancer: Strategies for overcoming resistance to chemotherapy. Nat Rev Cancer 3: 502-516, 2003.

5. Ozols RF, Bundy BN, Greer BE, Fowler JM, Clarke-Pearson D, Burger RA, Mannel RS, DeGeest K, Hartenbach EM and Baergen R; Gynecologic Oncology Group: Phase III trial of carboplatin and paclitaxel compared with cisplatin and paclitaxel in patients with optimally resected stage III ovarian cancer: A Gynecologic Oncology Group study. J Clin Oncol 21: 3194-3200, 2003.

6. du Bois A, Neijt JP and Thigpen JT: First line chemotherapy with carboplatin plus paclitaxel in advanced ovarian cancer-a new standard of care? Ann Oncol 10: (Suppl 1): S35-S41, 1999.

7. Biagi JJ and Eisenhauer EA: Systemic treatment policies in ovarian cancer: The next 10 years. Int J Gynecol Cancer 13 (Suppl 2): S231-S240, 2003.

8. Neijt JP, Engelholm SA, Tuxen MK, Sorensen PG, Hansen M, Sessa C, de Swart CA, Hirsch FR, Lund B and van Houwelingen HC: Exploratory phase III study of paclitaxel and cisplatin versus paclitaxel and carboplatin in advanced ovarian cancer. J Clin Oncol 18: 3084-3092, 2000

9. Kelland L: The resurgence of platinum-based cancer chemotherapy. Nat Rev Cancer 7: 573-584, 2007.

10. Siddik ZH: Cisplatin: Mode of cytotoxic action and molecular basis of resistance. Oncogene 22: 7265-7279, 2003.

11. Galluzzi L, Senovilla L, Vitale I, Michels J, Martins I, Kepp O, Castedo M and Kroemer G: Molecular mechanisms of cisplatin resistance. Oncogene 31: 1869-1883, 2012.

12. Greenlee RT, Hill-Harmon MB, Murray T and Thun M: Cancer statistics, 2001. CA Cancer J Clin 51: 15-36, 2001.
13. Gore ME, Fryatt I, Wiltshaw E and Dawson T: Treatment of relapsed carcinoma of the ovary with cisplatin or carboplatin following initial treatment with these compounds. Gynecol Oncol 36: 207-211, 1990.

14. Malz M, Weber A, Singer S, Riehmer V, Bissinger M, Riener MO, Longerich T, Soll C, Vogel A, Angel P, et al: Overexpression of far upstream element binding proteins: A mechanism regulating proliferation and migration in liver cancer cells. Hepatology 50: $1130-1139,2009$

15. Rabenhorst U, Beinoraviciute-Kellner R, Brezniceanu ML, Joos S, Devens F, Lichter P, Rieker RJ, Trojan J, Chung HJ, Levens DL and Zörnig M: Overexpression of the far upstream element binding protein 1 in hepatocellular carcinoma is required for tumor growth. Hepatology 50: 1121-1129, 2009

16. Pénzváltó Z, Lánczky A, Lénárt J, Meggyesházi N, Krenács T, Szoboszlai N, Denkert C, Pete I and Győrffy B: MEK1 is associated with carboplatin resistance and is a prognostic biomarker in epithelial ovarian cancer. BMC Cancer 14: 837, 2014.

17. Chung HJ and Levens D: c-myc expression: keep the noise down! Mol Cells 20: 157-166, 2005.

18. Duncan R, Bazar L, Michelotti G, Tomonaga T, Krutzsch H, Avigan M and Levens D: A sequence-specific, single-strand binding protein activates the far upstream element of c-myc and defines a new DNA-binding motif. Genes Dev 8: 465-480, 1994.

19. Liu J, Chung HJ, Vogt M, Jin Y, Malide D, He L, Dundr M and Levens D: JTV1 co-activates FBP to induce USP29 transcription and stabilize p53 in response to oxidative stress. EMBO J 30: 846-858, 2011.

20. Rabenhorst U, Thalheimer FB, Gerlach K, Kijonka M, Böhm S, Krause DS, Vauti F, Arnold HH, Schroeder T, Schnütgen F, et al: Single-Stranded DNA-Binding Transcriptional Regulator FUBP1 Is Essential for Fetal and Adult Hematopoietic Stem Cell Self-Renewal. Cell Rep 11: 1847-1855, 2015.

21. Dixit U, Liu Z, Pandey AK, Kothari R and Pandey VN: Fuse binding protein antagonizes the transcription activity of tumor suppressor protein p53. BMC Cancer 14: 925, 2014.

22. Dixit U, Pandey AK, Liu Z, Kumar S, Neiditch MB, Klein KM and Pandey VN: FUSE Binding Protein 1 facilitates persistent hepatitis $\mathrm{C}$ virus replication in hepatoma cells by regulating tumor suppressor p53. J Virol 89: 7905-7921, 2015.

23. Jameson GS, Petricoin EF, Sachdev J, Liotta LA, Loesch DM, Anthony SP, Chadha MK, Wulfkuhle JD, Gallagher RI, Reeder KA, et al: A pilot study utilizing multi-omic molecular profiling to find potential targets and select individualized treatments for patients with previously treated metastatic breast cancer. Breast Cancer Res Treat 147: 579-588, 2014.

24. Rask K, Nilsson A, Brännström M, Carlsson P, Hellberg P, Janson PO, Hedin L and Sundfeldt K: Wnt-signalling pathway in ovarian epithelial tumours: Increased expression of beta-catenin and GSK3beta. Br J Cancer 89: 1298-1304, 2003.

25. Barghout SH, Zepeda N, Xu Z, Steed H, Lee CH and Fu Y: Elevated $\beta$-catenin activity contributes to carboplatin resistance in A2780cp ovarian cancer cells. Biochem Biophys Res Commun 468: 173-178, 2015.

26. Murphy G and Nagase H: Localizing matrix metalloproteinase activities in the pericellular environment. FEBS J 278: 2-15, 2011.

27. Zitka O, Kukacka J, Krizkova S, Huska D, Adam V, Masarik M, Prusa R and Kizek R: Matrix metalloproteinases. Curr Med Chem 17: 3751-3768, 2010.

28. Butler GS and Overall CM: Updated biological roles for matrix metalloproteinases and new 'intracellular' substrates revealed by degradomics. Biochemistry 48: 10830-10845, 2009.

29. Rodríguez D, Morrison CJ and Overall CM: Matrix metalloproteinases: What do they not do? New substrates and biological roles identified by murine models and proteomics. Biochim Biophys Acta 1803: 39-54, 2010.

30. Kessenbrock K, Plaks V and Werb Z: Matrix metalloproteinases: Regulators of the tumor microenvironment. Cell 141: 52-67, 2010.

31. Hadler-Olsen E, Fadnes B, Sylte I, Uhlin-Hansen L and Winberg JO: Regulation of matrix metalloproteinase activity in health and disease. FEBS J 278: 28-45, 2011.

32. Zhang $\mathrm{J}$ and Chen QM: Far upstream element binding protein 1: A commander of transcription, translation and beyond. Oncogene 32: 2907-2916, 2013.

33. Wang YY, Gu XL, Wang C, Wang H, Ni QC, Zhang CH, Yu XF, Yang LY, He ZX, Mao GX and Yang SY: The far-upstream element-binding protein 2 is correlated with proliferation and doxorubicin resistance in human breast cancer cell lines. Tumour Biol 37: 9755-9769, 2016. 